\title{
Glutathione peroxidase 3 is a protective factor against acetaminophen-induced hepatotoxicity in vivo and in vitro
}

\author{
SYU-ICHI KANNO, AYAKO TOMIZAWA, SHIN YOMOGIDA and AKIYOSHI HARA \\ Department of Clinical Pharmacotherapeutics, Tohoku Medical and \\ Pharmaceutical University, Aoba-ku, Sendai 981-8558, Japan
}

Received January 11, 2017; Accepted June 6, 2017

DOI: $10.3892 /$ ijmm.2017.3049

\begin{abstract}
Acetaminophen (APAP) is a widely available antipyretic and analgesic; however, overdose of the drug inflicts severe damage to the liver. It is well established that the hepatotoxicity of APAP is initiated by formation of a reactive metabolite, $N$-acetyl- $p$-benzoquinone imine (NAPQI), which can be detoxified by conjugation with reduced glutathione (GSH), a typical antioxidant. We recently found that the blood mRNA expression level of glutathione peroxidase 3 (Gpx3), which catalyzes the oxidation of GSH, is associated with the extent of APAP-induced hepatotoxicity in mice. The present study was carried out to determine the in vivo and in vitro role of GPx3 in APAP-induced hepatotoxicity. In in vivo experiments, oral administration of APAP to mice induced liver injury. Such liver injury was greater in males than in females, although no gender difference in the plasma concentration of APAP was found. Female mice had a 2-fold higher expression of Gpx3 mRNA and higher plasma GPx activity than male mice. $17 \beta$-estradiol, a major female hormone, decreased APAP-induced hepatotoxicity and increased both the expression of blood Gpx3 mRNA and plasma GPx activity, suggesting that the cytoprotective action of this hormone is mediated by the increase in GPx3. To further clarify the role of GPx3 in APAP-induced hepatotoxicity, we evaluated the effect of a change in cellular GPx3 expression resulting from transfection of either siRNA-GPx3 or a GPx3 expression vector on NAPQI-induced cellular injury (as assessed by a tetrazolium assay) in in vitro experiments using heterogeneous cultured human cell lines (Huh-7 or K562). NAPQI-induced cell death was reduced by increased GPx3 and was enhanced by decreased GPx3. These results suggest that GPx3 is an important factor for inhibition of APAP-induced hepatotoxicity both in vivo and in vitro. To our knowledge, this
\end{abstract}

Correspondence to: Dr Syu-Ichi Kanno, Department of Clinical Pharmacotherapeutics, Tohoku Medical and Pharmaceutical University, 4-4-1 Komatsushima, Aoba-ku, Sendai 981-8558, Japan

E-mail: syu-kan@tohoku-mpu.ac.jp

Keywords: glutathione peroxidase 3, acetaminophen, hepatotoxicity, gender difference, $17 \beta$-estradiol, $N$-acetyl- $p$-benzoquinone imine is the first report to show a hepatoprotective role of cellular GPx3 against APAP-induced liver damage.

\section{Introduction}

Reduced glutathione (GSH) and its related enzymes constitute the key antioxidant system in the body for counteraction of oxidative stress injury (1). Among these enzymes, glutathione peroxidase (GPx) and its eight GPx isozymes (GPx1-8) that have been identified to date in mammals (2) can detoxify reactive oxygen species (ROS), including hydrogen peroxide and free radicals, in the presence of GSH. GPx3, which accounts for more than $97 \%$ of all plasma selenium in mice (3), has been shown to be upregulated in alcohol-induced hepatic injury to protect the liver from oxidative stress (4). According to a study on GPx gender differences, both GPx activities and GPx3 concentrations in serum are higher in females than in males (5).

Acetaminophen (APAP) is a widely available antipyretic and analgesic drug; however, its overdose can inflict severe damage on the liver by formation of its reactive metabolite, $N$-acetyl- $p$-benzoquinone imine (NAPQI). NAPQI can usually be detoxified by GSH, but its excess causes depletion of GSH, leading to production of ROS, and hence liver injury (6). It is noteworthy that males are more susceptible to APAP-induced liver injury than females, suggesting the resistance of females to APAP hepatotoxicity (7-12). This difference between genders is likely to be due to gender-dependent activities of GPX in mouse liver (13), although the involvement of gender differences in the levels and/or activities of other antioxidant-related enzymes, such as glutathione-S-transferase $\pi(7)$ and glutamate-cysteine ligase (8), cannot be ignored. Indeed, animals overexpressing plasma GPx have strong resistance to APAP-induced hepatotoxicity (13). We have recently shown in mice that, among isozymes of GPx, the degree of APAP-induced hepatotoxicity depends on the mRNA expression levels of GPX3 in the blood (14). This finding therefore raises the question as to whether the gender difference in the GPx3 level contributes to the difference in APAP-induced hepatotoxicity between genders. In association with the resistance to APAP in females, $17 \beta$-estradiol, a major female hormone, specifically attenuates acute hepatic damage and decreases mortality in APAP-overdosed male mice (15). Expression of the GPx3 gene is sensitive to circulating estrogens in skeletal muscle (16). A second question, therefore, is whether the hepatoprotective 
action of $17 \beta$-estradiol on APAP toxicity is mediated by increased synthesis of GPx3.

To answer these questions, the present study was carried out to determine the role of the GPx3 protein in APAP-induced hepatotoxicity in vivo and in vitro. In in vivo experiments in mice, we examined whether the degree of the GPx3 level accounts for the gender differences in APAP-induced hepatic injury, and whether GPx3 mediates the 17 $\beta$-estradiol reduction in APAP-induced hepatotoxicity. In in vitro experiments, to better clarify the role of cellular GPx3 in APAP-induced injury, we evaluated NAPQI-induced cellular injury when cellular GPx3 expression was altered by either transfected GPx3 siRNA or a transfected GPx3 expression vector.

\section{Materials and methods}

Animals and chemicals. Four-week-old adult male or female ddY mice (20-25 g) were obtained from Japan SLC Inc. (Hamamatsu, Japan). The animals were maintained on a 12-h light/dark cycle in a temperature- and humidity-controlled room. The experiments were conducted in accordance with the standards established by the Japanese Pharmacological Society and were approved by the Tohoku Medical and Pharmaceutical University of Institutional Animal Care and Use Committee (experimental no. 16014). The animals were allowed free access to laboratory pellet chow (CE-2; Clea Japan, Inc., Tokyo, Japan) and water before the experiments. APAP was purchased from Junsei Chemical Co., Ltd. (Nagano, Japan). 17 $\beta$-estradiol was obtained from Sigma Chemical Co. (St. Louis, MO, USA). NAPQI was purchased from Toronto Research Chemicals Inc. (Toronto, Canada). All other reagents, unless stated, were of the highest grade available and were supplied by either Sigma or Wako Pure Chemical Industries, Ltd. (Osaka, Japan).

RNA isolation and reverse transcription-quantitative polymerase chain reaction $(R T-q P C R)$ assay. The expression levels of mRNA were quantified using RT-qPCR according to our previously described methods (14). The animals were acclimated for 5-7 days prior to a pre-dose blood draw. Blood was drawn from the tail vein of each mouse into tubes containing $1 \mathrm{mM}$ EDTA as an anticoagulant. Total RNA was isolated using the ISOGEN reagent (Nippon Gene Co., Ltd., Tokyo, Japan), and RNA concentrations were determined using the NanoDrop 1000 (Thermo Fisher Scientific, Waltham, MA, USA). Total RNA from each sample $(0.1 \mu \mathrm{g})$ was reverse transcribed into single-stranded cDNA using the ReverTra Ace kit (Toyobo Co., Ltd., Osaka, Japan). Aliquots of the resulting cDNA preparations were then subjected to qPCR analysis using the KOD SYBR ${ }^{\circledR}$ qPCR Mix (Toyobo Co., Ltd.). A CFX Connect ${ }^{\mathrm{TM}}$ Real-Time PCR system (Bio-Rad Laboratories, Inc., Hercules, CA, USA) was used to determine mRNA expression levels of the GPx3 gene (GenBank accession no. NM_008161.3). The mRNA level was normalized against that of the GAPDH-encoding locus (GenBank accession no. NM_001289726). The sequences of the primer pairs used were obtained from the Takara Perfect Real Time Primers (Takara Bio, Shiga, Japan). The results of all assays were checked against melting curves in order to confirm the presence of single PCR products. At least two independent experiments were conducted and samples were assessed in (at least) triplicate in each experiment.

APAP hepatotoxicity and plasma concentration. Mice were orally administered (p.o.) APAP $(500 \mathrm{mg} / \mathrm{kg}$ in $10 \mathrm{ml} / \mathrm{kg}$ saline) at 18:00 h, and blood was collected $18 \mathrm{~h}$ later for determination of the serum activity of alanine aminotransferase (ALT) and aspartate aminotransferase (AST) using a colorimetric kit (Wako, Tokyo, Japan) as described in our previous reports $(17,18)$. Male mice were pretreated with $17 \beta$-estradiol at a dose of $0.2 \mathrm{mg} / \mathrm{kg}$ that was administered by intraperitoneal (i.p.) injection $4 \mathrm{~h}$ before treatment with APAP. The plasma concentration of APAP was measured using a modification of the method of Hori et al (19). Briefly, an aliquot (100 $\mu \mathrm{l})$ of plasma was diluted with an equal volume of $0.2 \mathrm{M} \mathrm{Na}_{2} \mathrm{HPO}_{4}$ and $0.1 \mathrm{M}$ citric acid buffer ( $\mathrm{pH} 3.0$ ), and the mixture then was combined with $300 \mu \mathrm{l}$ of ethyl acetate. The resulting samples were centrifuged at 3,000 $\mathrm{x}$ g for $5 \mathrm{~min}$ and the upper layer was retained and the lower layer was discarded. After the removal of solvents by evaporation under nitrogen gas, the sample was dissolved in $100 \mu \mathrm{l}$ of the mobile phase (methanol and $1 \mathrm{~N}$ acetic acid at a 70:30 ratio, v/v), and injected into a high-performance liquid chromatography system consisting of an Alliance 2695 Separations Module (Waters, Milford, MA, USA). The flow rate was kept constant at a rate of $0.4 \mathrm{ml} / \mathrm{min}$, and peaks were monitored at a wavelength of $254 \mathrm{~nm}$ for $10 \mathrm{~min}$. The concentration of APAP was calculated using an APAP standard curve.

GPx activity. Mouse plasma GPx was measured using a Glutathione Peroxidase Assay kit (catalog no. 703102; Cayman Chemical Co., Ann Arbor, MI, USA). The plasma samples were collected from a cut of the tail vein on the day before APAP treatment. The GPx level in each plasma sample $(20 \mu \mathrm{l})$ was analyzed according to the protocol supplied by the manufacturer of the kit.

Cell culture. The Huh-7 human liver cancer cell line and the K562 human erythroleukemia cell line were supplied by the Cell Resource Center for Biomedical Research, Tohoku University (Sendai, Japan). The cells were maintained in RPMI-1640 medium supplemented with $10 \%$ fetal bovine serum, $100 \mathrm{U} / \mathrm{ml}$ penicillin $\mathrm{G}$, and $100 \mu \mathrm{g} / \mathrm{ml}$ streptomycin at $37^{\circ} \mathrm{C}$ in a humidified $5 \% \mathrm{CO}_{2}-95 \%$ air incubator under standard conditions. The cells were counted, excluding cells stained with $0.2 \%$ Trypan blue. To maintain exponential growth, cells were seeded at a density of $5 \times 10^{4}$ cells $/ \mathrm{ml}$ and were passaged every 3-4 days. Cells were cultured in $2 \mathrm{ml}$ aliquots in $35-\mathrm{mm}$ dishes for other assays.

Cell survival assay. Cellular survival was assessed using the water-soluble tetrazolium WST-1 (sodium 5-(2,4-disulfophe nyl)-2-(4-iodophenyl)-3-(4-nitrophenyl)-2H tetrazolium inner salt) assay, which detects metabolically competent cells with an intact mitochondrial electron transport chain (20). Briefly, $1 \times 10^{4}$ cells were seeded into 96-well plates and cultured overnight. The cells were incubated with NAPQI for the indicated times, and medium containing the WST-1 solution (0.5 mM WST-1 and $0.02 \mathrm{mM}$ 1-methoxy-5-methylphenazinium methylsulfate; 1-PMS) was added to each well. The cells were incubated for $60 \mathrm{~min}$ at $37^{\circ} \mathrm{C}$, and absorption at a wavelength of $438 \mathrm{~nm}$ 
(ref. $620 \mathrm{~nm}$ ) was measured using a SH-1200 Microplate Reader $^{\circledR}$ (Corona, Hitachinaka, Japan). Control cells were treated with $0.1 \%$ DMSO. Cell viability was calculated using the following formula: Absorbance in the treated sample/absorbance in the control x100 (\%).

GPx3 knockdown. siRNA-GPx3 (siGPx3) and siRNA-control [non-targeting siRNA; negative control (Neg)] were transfected into Huh-7 or K562 cells using HyperFect transfection reagent (Qiagen, Inc., Valencia, CA, USA) according to the protocol supplied by the manufacturer. A non-targeting siRNA was used as a control for the non-sequence-specific effects of the transfected siRNAs. The siRNAs used were siGPx3, a Silencer ${ }^{\circledR}$ Select Pre-designed siRNA Product (ID no. s6109; Ambion, Austin, TX, USA), and negative control siRNA from AllStars Neg. Control siRNA (ID no. AM4611; Qiagen, Inc.). Briefly, $5 \times 10^{4}$ cells containing each siRNA (final concentration, $10 \mathrm{nM}$ ) and the HyperFect reagent were incubated for $24 \mathrm{~h}$ for assessment of GPx3 expression or cytotoxic effects induced by NAPQI.

GPx3 overexpression. The cells were transfected with a GFP-tagged ORF clone of GPx 3 cloned into a pCMV6-AC-GFP vector (OriGene Technologies, Inc., Rockville, MD, USA). Plasmid DNA was transfected into Huh-7 or K562 cells using ViaFect ${ }^{\mathrm{TM}}$ Transfection Reagent (Promega Corp., Madison, WI, USA) and the Neon ${ }^{\mathrm{TM}}$ Transfection System (Invitrogen Life Technologies, Carlsbad, CA, USA), respectively, according to the instructions provided by the manufacturer. The presence of the transfected vector in the cells was confirmed by fluorescence microscopic observation (488 nm, GFP fluorescence) as previously described (21).

Western blot analysis. The cells were washed with phosphate buffered saline (PBS) and lysed in CelLytic $\mathbf{M}^{\circledR}$ (Sigma-Aldrich, St. Louis, MO, USA) to collect a total cell lysate according to the manufacturer's instructions. Protein concentration was measured using the $\mathrm{BCA}^{\mathrm{TM}}$ protein assay kit (Thermo Fisher Scientific, Inc., Rockford, IL, USA) according to the instructions provided by the manufacturer. Following electrophoreses of protein samples $(30 \mu \mathrm{g})$ on a $10 \%$ SDS-polyacrylamide gel, the protein was transferred to a polyvinylidene difluoride membrane. The membrane was blocked with Blocking One ${ }^{\circledR}$ (Nacalai Tesque, Inc., Kyoto, Japan) for $1 \mathrm{~h}$ and then incubated with a primary antibody overnight at $4^{\circ} \mathrm{C}$. Antibodies against human GPx3 (mouse monoclonal; ab27325; Abcam, Cambridge, MA, USA) and against $\beta$-actin as the loading control (rabbit polyclonal; \#4967; Cell Signaling Technology, Inc., Danvers, MA, USA) were used. The membrane was then washed with wash buffer (PBS containing 0.05\% Tween-20) and incubated with horseradish peroxidase-linked secondary antibody [anti-mouse IgG (\#7076) or anti-rabbit IgG (\#7074); Cell Signaling Technology, Inc.] for $1 \mathrm{~h}$. After another wash with wash buffer, protein signals were analyzed by enhanced chemiluminescence with the Pierce ${ }^{\circledR}$ Western Blotting substrate (Thermo Fisher Scientific, Inc.).

Statistical analysis. Statistical analysis was performed with two-way analysis of variance, followed by the Bonferroni test to compare among multiple groups. Data are expressed as means \pm standard error of the mean $(\mathrm{SEM}) . \mathrm{P}<0.05$ was considered to indicate a statistically significant difference. Statistical analyses were performed using Ekuseru-Toukei 2012 software (Social Survey Research Information Co., Ltd., Tokyo, Japan).

\section{Results}

Female mice are resistant to APAP hepatotoxicity and show a higher GPX3 activity. There was no significant difference in serum activities of AST and ALT, which are markers of liver injury, between male and female mice before APAP treatment. These values were $135 \pm 28 \mathrm{KU}$ and $48 \pm 8 \mathrm{KU}$, respectively, in male mice, and $104 \pm 18 \mathrm{KU}$ and $38 \pm 11 \mathrm{KU}$, respectively, in female mice. Compared to these basal values, the serum activities of AST and ALT after oral administration of APAP were increased 10- and 31-fold, respectively in male mice, whereas they were only increased 3-and 4-fold, respectively in female mice (Fig. 1A and B). These results suggested that APAP inflicted damage to the liver of the mice (ddY mice), and that sensitivity to APAP hepatotoxicity was much lower in females than in males. It was noted, however, that the plasma APAP concentration after the treatment was not significantly different between the male and female mice (Fig. 1C). Female mice also showed a slight but significantly higher activity of plasma GPx (Fig. 1E), and a 2-fold higher expression of Gpx3 mRNA (Fig. 1D), relative to the male mice, suggesting higher activity of this antioxidant-related enzyme in females.

$17 \beta$-estradiol attenuates APAP-induced hepatotoxicity and increases GPX3 in male mice. We examined the effect of $17 \beta$-estradiol on APAP-induced hepatotoxicity in male mice. In a preliminary experiment, pretreatment with $17 \beta$-estradiol did not influence plasma APAP concentrations (data not shown). As shown in Fig. 2A and B, serum aminotransferase activities in the group treated with a combination of $17 \beta$-estradiol and APAP were much lower than those in the group treated with APAP alone, suggesting a protective action of $17 \beta$-estradiol against APAP-induced hepatotoxicity. The blood level of Gpx3 mRNA expression was approximately 2-fold higher (Fig. 2C) and the activities of GPx in plasma were marginally significantly elevated (Fig. 2D) in the 17 $\beta$-estradiol-treated group compared with the control group, suggesting that $17 \beta$-estradiol enhances the activities of the antioxidant-related enzyme in males.

NAPQI-induced reduction in cell survival is affected by cellular GPx3 expression. To determine the effects of GPx3 on APAP-induced toxicity at the cellular level, in vitro experiments were performed with heterogeneous cultured human cell lines (the human hepatoma Huh-7 cells and the human erythroleukemia K562 cells). The extent of cell injury in response to various concentrations of NAPQI, as judged by analysis of cell survival, is shown in Fig. 3A and B. NAPQI reduced the survival of both Huh-7 and K562 cell lines in a concentration- and time-dependent manner. The amount of NAPQI required to inhibit cell survival was higher in the Huh-7 cells than this amount in the K562 cells; e.g., the 50\% inhibitory concentrations $\left(\mathrm{IC}_{50}\right)$ at $72 \mathrm{~h}$ of incubation were calculated as 35.5 and $5.4 \mu \mathrm{M}$, respectively, indicating that the NAPQI sensitivity was 6.6-fold lower in Huh-7 cells than that noted in the K562 cells. 
A

AST

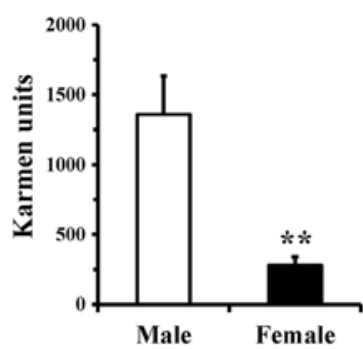

B

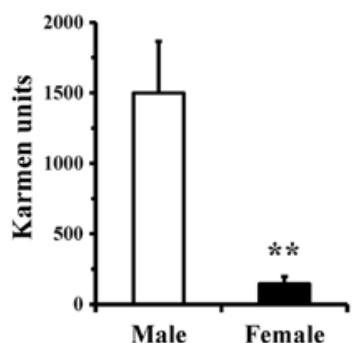

C
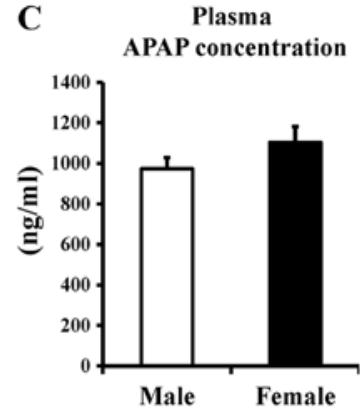

D

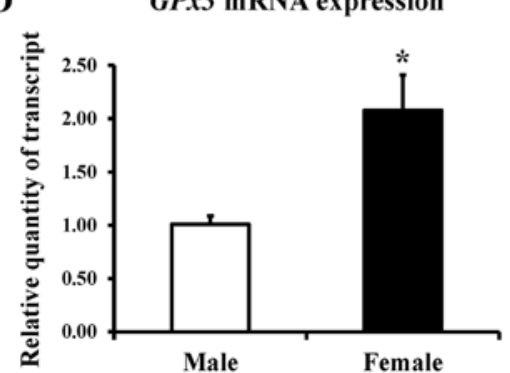

$\mathbf{E}$

Figure 1. Comparison of APAP-induced hepatotoxicity, plasma APAP concentration, blood GPx3 mRNA expression, and plasma GPx activity between male and female ddY mice. Hepatotoxicity was determined by measuring the serum activities of (A) AST and (B) ALT at $18 \mathrm{~h}$ after oral administration of APAP $(500 \mathrm{mg} / \mathrm{kg}$ ). (C) Plasma APAP concentration, (D) blood GPx3 mRNA expression, and (E) plasma GPx activity were measured as described in the 'Materials and methods' section. Data are expressed as means \pm SEM. ${ }^{*} \mathrm{P}<0.05,{ }^{* * *} \mathrm{P}<0.01$ compared to male mice ( $\mathrm{n}=80$ for each mouse group). APAP, acetaminophen; AST, aspartate aminotransferase; ALT, alanine aminotransferase.
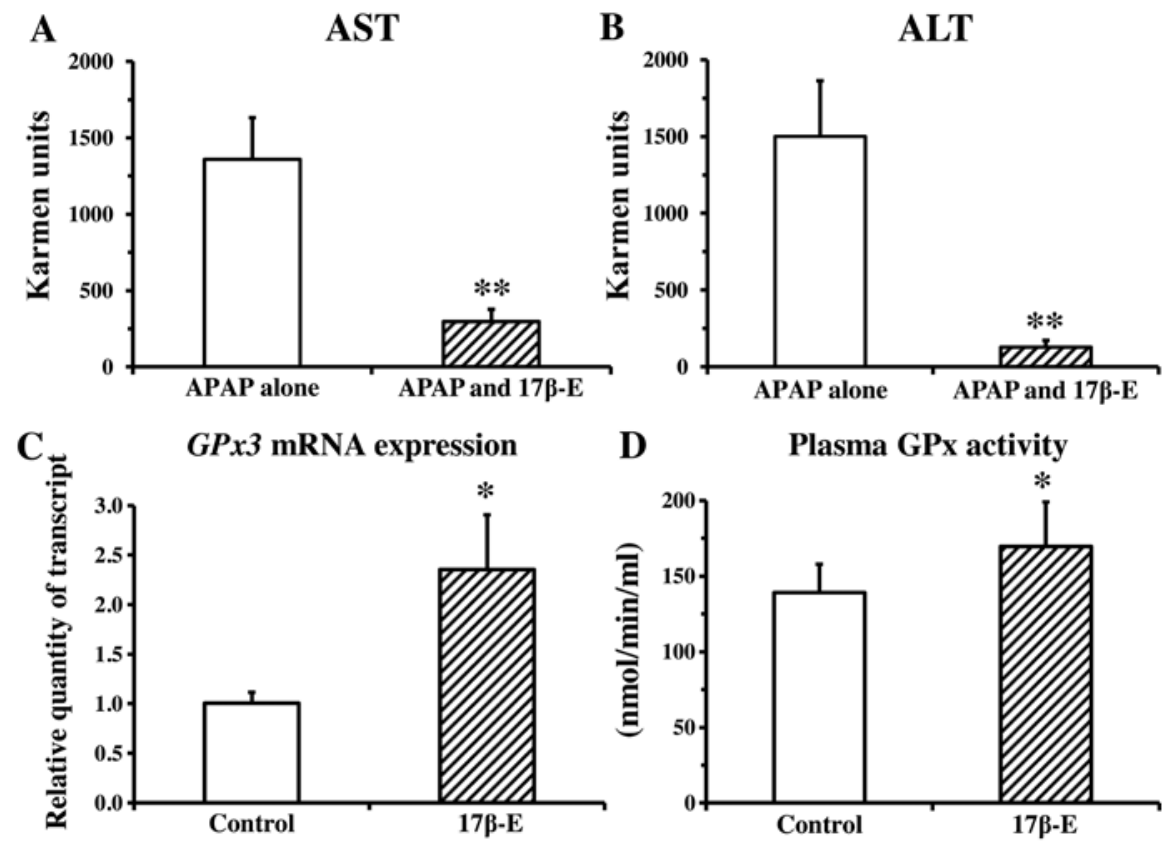

Figure 2. Effects of $17 \beta$-estradiol on APAP-induced hepatotoxicity, blood GPx3 mRNA expression, and plasma GPx activity in male mice. Hepatotoxicity was determined by measuring the serum activities of (A) AST and (B) ALT at $18 \mathrm{~h}$ after oral administration of APAP (500 mg/kg) to male mice with and without $17 \beta$-estradiol (17 $\beta$-E at $0.2 \mathrm{mg} / \mathrm{kg}$, i.p.) pretreatment. (C) Blood GPx3 mRNA expression, and (D) plasma GPx activity were measured as described in the 'Materials and methods' section. Data are expressed as means \pm SEM. ${ }^{*} \mathrm{P}<0.05,{ }^{* *} \mathrm{P}<0.01$ compared to APAP alone or control group ( $\mathrm{n}=40$ for each mouse group). APAP, acetaminophen; AST, aspartate aminotransferase; ALT, alanine aminotransferase; i.p., intraperitoneal.

We next examined the potential role of GPx3 in these effects of NAPQI by analysis of the effect of changes in cellular GPx3 expression brought about by transfection of siGPx3 or a GPx3 expression vector on the NAPQI-induced reduction of cell survival in the Huh-7 (Fig. 4) or K562 (Fig. 5) cell line.
After transfection of Huh-7 and K562 cells with siGPx3 for $24 \mathrm{~h}$, the expression level of GPx3 was barely detectable in either cell line compared to the control (non-transfected) group. The knockdown efficacy of GPx3 mRNA was estimated to be $>99 \%$ by RT-qPCR. The expression levels 
A

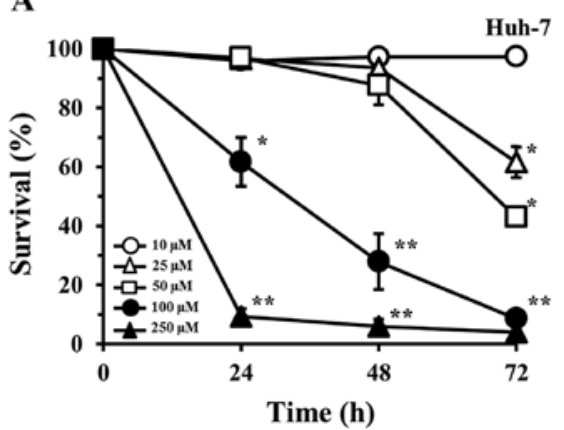

B

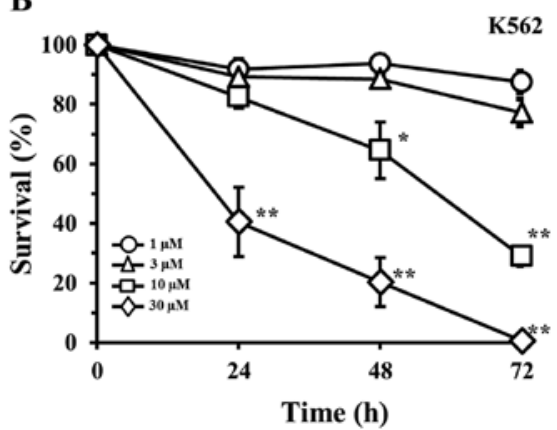

Figure 3. NAPQI-induced reduction in cell survival in the human liver cancer Huh-7 and human erythroleukemia K562 cell lines. (A) Huh-7 or (B) K562 cells were incubated with various concentrations of NAPQI for the indicated times, and the degree of cell survival was then assessed using the WST-1 assay. The percentage of surviving cells was calculated relative to the cell number of the respective NAPQI-untreated sample. Data are expressed as means \pm SEM of three samples. ${ }^{*} \mathrm{P}<0.05,{ }^{* * *} \mathrm{P}<0.01$ compared to before incubation. NAPQI, N-acetyl-p-benzoquinone imine; WST, water-soluble tetrazolium.

A

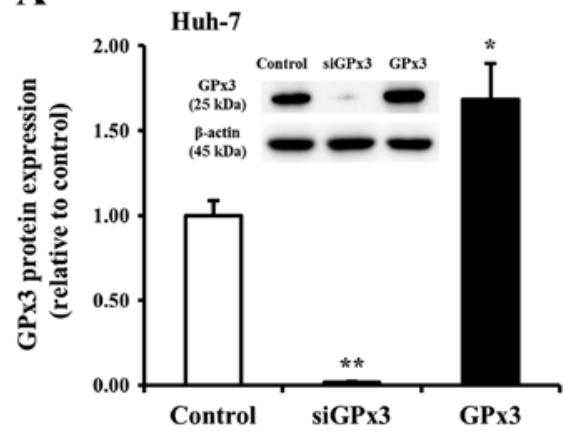

B

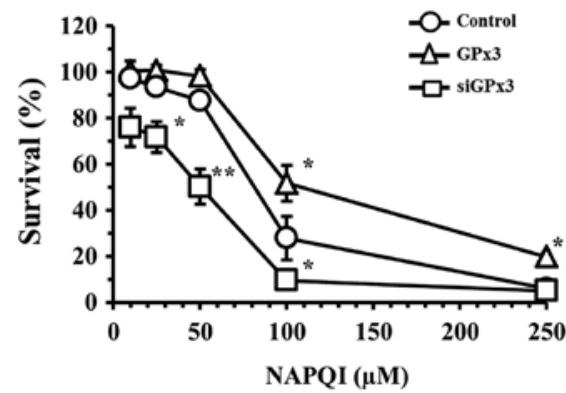

Figure 4. Effect of changes in cellular GPx 3 expression due to transfected siGPx 3 or a GPx 3 expression vector in Huh-7 cells on the degree of NAPQI-induced reduction of cell survival. (A) A representative graph of the quantification of the cellular levels of GPx3 protein expression detected by western blot analysis in control (non-transfected) and transfected Huh-7 cells. The cells were transfected with either siGPx3 for GPx3 knockdown, or with a GPx3 expression vector for GPx3 over-expression. Data are means \pm SEM of three samples. ${ }^{*} \mathrm{P}<0.05,{ }^{* *} \mathrm{P}<0.01$ compared to control group. (B) NAPQI-induced cytotoxicity assessed by WST-1 assay in Huh-7 cells transfected as in (A). The degree of survival of NAPQI-treated cells is expressed as a percentage of that in NAPQI-untreated control cells. The results are means \pm SEM of three samples. ${ }^{*} \mathrm{P}<0.05,{ }^{* *} \mathrm{P}<0.01$ compared to corresponding control cells. NAPQI, N-acetyl-p-benzoquinone imine; WST, water-soluble tetrazolium.

A

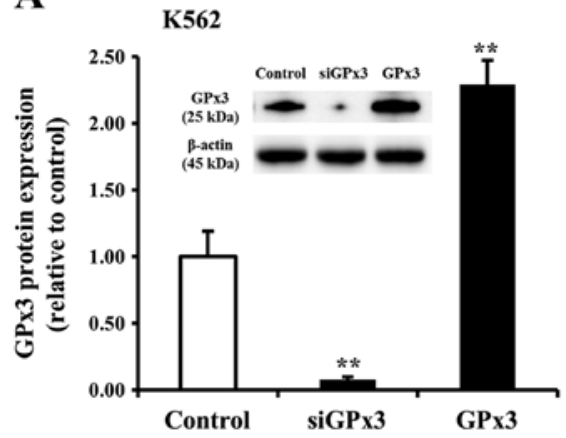

B

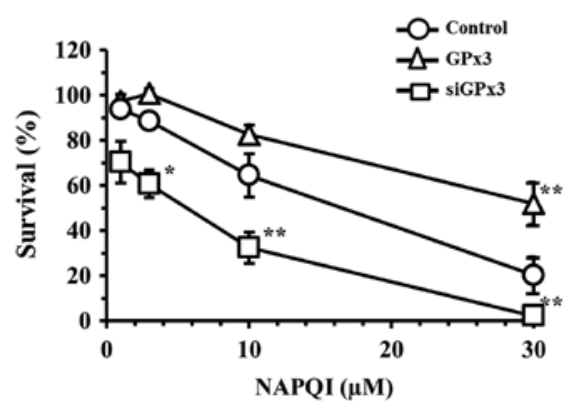

Figure 5. Effect of changes in cellular GPx3 expression due to transfected siGPx3 or a GPx3 expression vector in K562 cells on the degree of NAPQI-induced reduction of cell survival. (A) A representative graph of the quantification of the cellular levels of GPx3 protein expression detected by western blot analysis in control (non-transfected) and transfected K562 cells. The cells were transfected with either siGPx3 for GPx3 knockdown, or with a GPx3 expression vector for GPx 3 over-expression. Data are means \pm SEM of three samples. ${ }^{*} \mathrm{P}<0.05,{ }^{* *} \mathrm{P}<0.01$ compared to control group. (B) NAPQI-induced cytotoxicity assessed by WST-1 assay in K562 cells transfected as in (A). The degree of survival of NAPQI-treated cells is expressed as a percentage of that in NAPQI-untreated control cells. The results are means \pm SEM of three samples. ${ }^{*} \mathrm{P}<0.05,{ }^{* *} \mathrm{P}<0.01$ compared to corresponding control cells. NAPQI, N-acetyl-p-benzoquinone imine; WST, water-soluble tetrazolium.

of GPx3 in the GPx3 vector-transfected group were 1.7-fold and 2.3-fold higher in the Huh-7 and K562 cells, respectively, than that in the respective non-transfected control group (Figs. 4A and 5A). In the non-transfected control Huh-7 group, 
incubation (for $48 \mathrm{~h}$ ) with NAPQI reduced cell survival to $97,94,88,28$, and $6 \%$ of the control at a concentration of $10,25,50,100$, and $250 \mu \mathrm{M}$, respectively (Fig. 4B). In the non-transfected control K562 group, incubation (for $48 \mathrm{~h}$ ) with NAPQI reduced cell survival to $94,89,65$, and $20 \%$ of the control at a concentration of $1,3,10$, and $30 \mu \mathrm{M}$ of NAPQI, respectively (Fig. 5B). The reduction in cell survival by NAPQI was potentiated in each siGPx3-transfected group, but was inhibited in each GPx3-transfected group (GPx3 overexpression group) vs. the respective non-treated control in both cell lines (Figs. 4B and 5B). In a preliminary experiment, neither transfection with control siRNA nor with vector alone affected cell survival of either cell line at incubation times of $72 \mathrm{~h}$ or shorter. These results suggested that GPx3 has a protective role against NAPQI-induced cellular injury in both Huh-7 and K562 cells.

\section{Discussion}

The present study demonstrated that APAP caused liver injury in vivo, as assessed by increases in serum AST and ALT levels in ddY mice, and that females were less sensitive to APAP hepatotoxicity than males (Fig. 1). Resistance of females to APAP-induced hepatotoxicity has also been observed in another mouse species, C57BL/6 mice $(7,9,10)$. These findings suggest that APAP induces gender-dependent but species-independent liver injury in mice. It is well established that there is a gender difference in the activities of liver metabolic enzymes, which are involved in metabolism and excretion of drugs. However, the gender difference in APAP-induced hepatotoxicity is not likely to be due to a difference in metabolism and excretion of this drug, since the concentrations of free APAP in bile, urine, or serum were not reported to be different between male and female mice (7). We also confirmed that there was no difference in the plasma concentration of APAP between genders. It is unlikely, therefore, that the resistance to APAP-induced hepatotoxicity in females is due to the difference in APAP pharmacokinetics between genders.

It is well established that APAP hepatotoxicity is initiated by the formation of NAPQI, which causes depletion of GSH, leading to production of ROS, and hence hepatic injury. Some previous reports have indicated that the activity of glutamate-cysteine ligase, a rate-limiting enzyme in GSH synthesis, is higher in female mice than in male mice, and therefore early recovery of hepatic GSH may confer resistance to APAP-induced liver injury. These findings suggest that gender differences in APAP hepatotoxicity can be attributed to the activity of glutamate-cysteine ligase (8-11). Other reports have shown that GSH amount or GSH-related enzymatic activity in mouse liver is involved in the gender difference in APAP hepatotoxicity $(7,8)$. The analysis of blood samples in the present study demonstrated that both blood GPx3 mRNA expression and plasma GPx activity (i.e., GPx3 activity) were higher in female mice than in male mice. Since GPx3 can protect some tissues including liver from oxidative stress by ROS, the resistance to APAP toxicity observed in female mice is thought to be due, at least in part, to higher GPx3 activity. These data suggested that endogenous estrogens may play a role in the resistance to APAP toxicity and the increased activity of GPx that was observed in female mice. In an in vivo experiment, we found that treatment of male mice with $17 \beta$-estradiol, which is one of the most active estrogens, markedly inhibited the APAP-induced increases in serum AST and ALT, and increased both Gpx3 mRNA expression and plasma GPx activity vs. the non-treated control mice. These data (Fig. 2) were comparable to those in female mice (Fig. 1). These findings strongly suggested that higher activity of GPx3 contributes to the beneficial action of $17 \beta$-estradiol and to the resistance of females against APAP hepatotoxicity. Another study demonstrated that $17 \beta$-estradiol is capable of attenuating APAP toxicity without a change in normal GSH levels in male liver (15). Thus, $17 \beta$-estradiol can protect the liver from APAP-induced toxicity, and the antioxidant activity of estrogen is attributed to its effect on powerful antioxidant enzymes in the whole body.

It should be noted, however, that estrogens exert antioxidant actions through a variety of mechanisms; they both reduce the production of ROS and upregulate a number of cellular antioxidative defense molecules (22-24). The antioxidant potency of estrogens, which is shared with other phenolic agents, is thought to be due to the presence of a benzene ring that scavenges hydroxyl radicals (25). On the other hand, the antioxidant effect of estrogens on rat hepatocytes is not dependent on the chemical structure of the estrogens per se, but rather on their hormonal effects (26). Within the cell, estrogens bind to their membrane-bound receptors, activate the MAP kinase-NFאB pathway, and increase transcription of antioxidant enzymes, especially GPx and superoxide dismutase (SOD) 2 (27). In addition, the activity of certain antioxidant enzymes including GPx is increased in lymphocytes treated with estrogen and its receptor agonists (28). These findings in biochemical and immune fields support the effect of estrogen to enhance the activity of antioxidant enzymes. Thus, the antioxidant effect of estrogens is likely to be due to both direct and indirect protective activities against ROS, although the detailed mechanisms remain unclear.

Intracellular overexpression of GPx can detoxify the final products of oxidative stress and is more efficient in protecting cells in vitro against ROS than SOD or catalase (29). To clarify the role of GPx in cell damage induced by NAPQI in vitro, we investigated whether NAPQI-induced cell death is altered by a change in cellular GPx3 expression brought about by transfection of either siGPx3 or a GPx3 expression vector into Huh-7 or K562 cells (Figs. 4 and 5). These experiments showed that NAPQI-induced cell death was reduced by increased GPx3 expression and was enhanced by decreased GPx3 expression, suggesting a crucial role for intracellular GPx in preventing the oxidative stress induced by NAPQI. The role of GPx in APAP-induced hepatotoxicity could be further clarified by analysis using knock-out mice of GPx. However, we did not perform this analysis, as there is no available knock-out in mouse strain (ddY) used in the present study. There is also previous evidence that shows that the antioxidant action of GPx contributes to inhibition of tumor progression and recurrence. The expression of GPx3 is downregulated within tumor tissues in several types of cancers (30-33). In addition, lower amounts of plasma GPx3 is correlated with tumor progression and recurrence in hepatocellular carcinoma (HCC) patients, and overexpression of GPx3 or administration of recombinant GPx3 inhibits the proliferation and invasiveness of HCC 
cells (34). In our experiments, there was no significant change in cell growth by transfection of siGPx 3 or a GPx3 expression vector during incubation for $72 \mathrm{~h}$ after transfection compared to the non-transfected or control-transfected cells. However, longer incubation could possibly change the growth of these transfected cells.

In conclusion, GPx3 is an important factor for inhibition of APAP-induced hepatotoxicity both in vivo and in vitro. To our knowledge, this is the first report to show a hepatoprotective role of cellular GPx3, although there have been many reports of the attenuation of APAP-induced liver injury by antioxidant enzymes. Nevertheless, more studies are required to determine why GPx3 transcription is regulated by estrogen or other factors and to investigate the mechanisms of resistance to APAP toxicity.

\section{Acknowledgements}

This study was supported in part by a Grant-in-Aid for Scientific Research (C) (KAKENHI 25460220) from the Japan Society for the Promotion of Science, and by a Matching Fund Subsidy for Private Universities from the Ministry of Education, Culture, Sports, Science and Technology of Japan.

\section{References}

1. Deneke SM and Fanburg BL: Regulation of cellular glutathione. Am J Physiol 257: L163-L173, 1989.

2. Brigelius-Flohé R and Maiorino M: Glutathione peroxidases. Biochim Biophys Acta 1830: 3289-3303, 2013.

3. Olson GE, Whitin JC, Hill KE, Winfrey VP, Motley AK, Austin LM, Deal J, Cohen HJ and Burk RF: Extracellular glutathione peroxidase (Gpx3) binds specifically to basement membranes of mouse renal cortex tubule cells. Am J Physiol Renal Physiol 298: F1244-F1253, 2010.

4. Li YG, Ji DF, Zhong S, Shi LG, Hu GY and Chen S: Saponins from Panax japonicus protect against alcohol-induced hepatic injury in mice by upregulating the expression of GPX3, SOD1 and SOD3. Alcohol Alcohol 45: 320-331, 2010.

5. Rush JW and Sandiford SD: Plasma glutathione peroxidase in healthy young adults: Influence of gender and physical activity. Clin Biochem 36: 345-351, 2003.

6. Nelson SD: Molecular mechanisms of the hepatotoxicity caused by acetaminophen. Semin Liver Dis 10: 267-278, 1990.

7. Dai G, He L, Chou N and Wan YJ: Acetaminophen metabolism does not contribute to gender difference in its hepatotoxicity in mouse. Toxicol Sci 92: 33-41, 2006.

8. Sheng Y, Liang Q, Deng Z, Ji L and Wang Z: Acetaminophen induced gender-dependent liver injury and the involvement of GCL and GPx. Drug Discov Ther 7: 78-83, 2013.

9. Botta D, Shi S, White CC, Dabrowski MJ, Keener CL, Srinouanprachanh SL, Farin FM, Ware CB, Ladiges WC, Pierce RH, et al: Acetaminophen-induced liver injury is attenuated in male glutamate-cysteine ligase transgenic mice. J Biol Chem 281: 28865-28875, 2006.

10. McConnachie LA, Mohar I, Hudson FN, Ware CB, Ladiges WC, Fernandez C, Chatterton-Kirchmeier S, White CC, Pierce RH and Kavanagh TJ: Glutamate cysteine ligase modifier subunit deficiency and gender as determinants of acetaminophen-induced hepatotoxicity in mice. Toxicol Sci 99: 628-636, 2007.

11. Masubuchi Y, Nakayama J and Watanabe Y: Sex difference in susceptibility to acetaminophen hepatotoxicity is reversed by buthionine sulfoximine. Toxicology 287: 54-60, 2011.

12. Du K, Williams CD, McGill MR and Jaeschke H: Lower susceptibility of female mice to acetaminophen hepatotoxicity: Role of mitochondrial glutathione, oxidant stress and c-jun N-terminal kinase. Toxicol Appl Pharmacol 281: 58-66, 2014.

13. Mirochnitchenko O, Weisbrot-Lefkowitz M, Reuhl K, Chen L, Yang $\mathrm{C}$ and Inouye $\mathrm{M}$ : Acetaminophen toxicity. Opposite effects of two forms of glutathione peroxidase. J Biol Chem 274 10349-10355, 1999.
14. Kanno S, Tomizawa A and Yomogida S: Detecting mRNA Predictors of acetaminophen-induced hepatotoxicity in mouse blood using quantitative real-time PCR. Biol Pharm Bull 39: 440-445, 2016.

15. Chandrasekaran VR, Periasamy S, Liu LL and Liu MY: $17 \beta$-Estradiol protects against acetaminophen-overdose-induced acute oxidative hepatic damage and increases the survival rate in mice. Steroids 76: 118-124, 2011.

16. Baltgalvis KA, Greising SM, Warren GL and Lowe DA: Estrogen regulates estrogen receptors and antioxidant gene expression in mouse skeletal muscle. PLoS One 5: e10164, 2010.

17. Kanno S, Ishikawa M, Takayanagi M, Takayanagi Y and Sasaki K: Potentiation of acetaminophen hepatotoxicity and mortality by doxapram in mice. Biol Pharm Bull 21: 934-937, 1998.

18. Kanno S, Tomizawa A, Hiura T, Osanai Y, Kakuta M, Kitajima Y, Koiwai K, Ohtake T, Ujibe M and Ishikawa M: Melatonin protects on toxicity by acetaminophen but not on pharmacological effects in mice. Biol Pharm Bull 29: 472-476, 2006.

19. Hori Y, Iwasaki Y, Kuroki Y, Komiyayama Y, Nakatani H and Namera A: [Practical analysis of toxic substances useful for clinical toxicology--4--Acetaminophen]. Chudoku Kenkyu 15: 385-390, 2002.

20. Berridge MV, Herst PM and Tan AS: Tetrazolium dyes as tools in cell biology: New insights into their cellular reduction. Biotechnol Annu Rev 11: 127-152, 2005.

21. Kanno S, Kurauchi K, Tomizawa A, Yomogida S and Ishikawa M: Pifithrin-alpha has a p53-independent cytoprotective effect on docosahexaenoic acid-induced cytotoxicity in human hepatocellular carcinoma HepG2 cells. Toxicol Lett 232: 393-402, 2015.

22. Huh K, Shin US, Choi JW and Lee SI: Effect of sex hormones on lipid peroxidation in rat liver. Arch Pharm Res 17: 109-114, 1994.

23. Gómez-Zubeldia MA, Hernandez R, Viguera J, Arbues JJ, Aparicio A and Millán JC: Effect of bilateral ovariectomy and ovarian steroid hormones on the antioxidant systems and plasma malondialdehyde levels in Wistar rats. Endocr Res 26: 97-107, 2000.

24. Kumar S, Lata K, Mukhopadhyay S and Mukherjee TK: Role of estrogen receptors in pro-oxidative and anti-oxidative actions of estrogens: A perspective. Biochim Biophys Acta 1800: 1127-1135, 2010.

25. Sugioka K, Shimosegawa $Y$ and Nakano M: Estrogens as natural antioxidants of membrane phospholipid peroxidation. FEBS Lett 210: 37-39, 1987.

26. Ruiz-Larrea MB, Leal AM, Martín C, Martínez R and Lacort M: Antioxidant action of estrogens in rat hepatocytes. Rev Esp Fisiol 53: 225-229, 1997.

27. Borrás C, Gambini J, Gómez-Cabrera MC, Sastre J, Pallardó FV, Mann GE and Viña J: 17beta-oestradiol up-regulates longevityrelated, antioxidant enzyme expression via the ERK1 and ERK2[MAPK]/NFkappaB cascade. Aging Cell 4: 113-118, 2005.

28. Priyanka HP, Krishnan HC, Singh RV, Hima L and Thyagarajan S: Estrogen modulates in vitro $\mathrm{T}$ cell responses in a concentrationand receptor-dependent manner: Effects on intracellular molecular targets and antioxidant enzymes. Mol Immunol 56: 328-339, 2013.

29. Michiels C, Raes M, Toussaint O and Remacle J: Importance of Se-glutathione peroxidase, catalase, and $\mathrm{Cu} / \mathrm{Zn}$-SOD for cell survival against oxidative stress. Free Radic Biol Med 17: 235-248, 1994.

30. Barrett CW, Ning W, Chen X, Smith JJ, Washington MK, Hill KE, Coburn LA, Peek RM, Chaturvedi R, Wilson KT, et al: Tumor suppressor function of the plasma glutathione peroxidase gpx3 in colitis-associated carcinoma. Cancer Res 73: 1245-1255, 2013.

31. He Y, Wang Y, Li P, Zhu S, Wang J and Zhang S: Identification of GPX3 epigenetically silenced by CpG methylation in human esophageal squamous cell carcinoma. Dig Dis Sci 56: 681-688, 2011.

32. Peng DF, Razvi M, Chen H, Washington K, Roessner A, Schneider-Stock R and El-Rifai W: DNA hypermethylation regulates the expression of members of the Mu-class glutathione $\mathrm{S}$-transferases and glutathione peroxidases in Barrett's adenocarcinoma. Gut 58: 5-15, 2009

33. Murawaki Y, Tsuchiya H, Kanbe T, Harada K, Yashima K, Nozaka K, Tanida O, Kohno M, Mukoyama T, Nishimuki E, et al: Aberrant expression of selenoproteins in the progression of colorectal cancer. Cancer Lett 259: 218-230, 2008.

34. Qi X, Ng KT, Lian QZ, Liu XB, Li CX, Geng W, Ling CC, Ma YY, Yeung WH, Tu WW, et al: Clinical significance and therapeutic value of glutathione peroxidase 3 (GPx3) in hepatocellular carcinoma. Oncotarget 5: 11103-11120, 2014. 